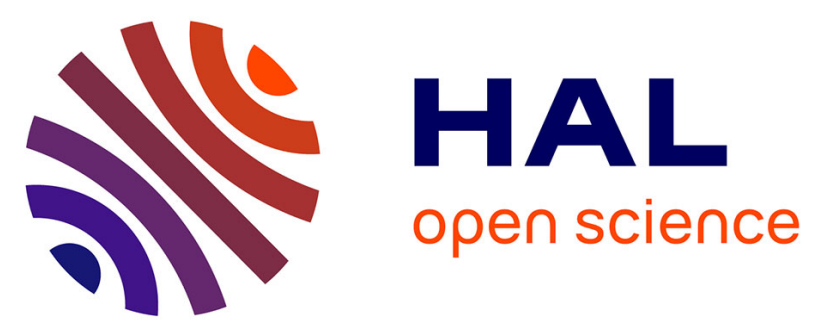

\title{
Evidence that ACETATE NON-UTILIZING 1 prevents carbon leakage from peroxisomes during lipid mobilization in Arabidopsis seedlings
}

Elizabeth Allen, Annick Moing, Jonathan Ad Wattis, Tony Larson, Mickael Maucourt, Ian A Graham, Dominique Rolin, Mark A Hooks

\section{To cite this version:}

Elizabeth Allen, Annick Moing, Jonathan Ad Wattis, Tony Larson, Mickael Maucourt, et al.. Evidence that ACETATE NON-UTILIZING 1 prevents carbon leakage from peroxisomes during lipid mobilization in Arabidopsis seedlings. Biochemical Journal, 2011, 437 (3), pp.505-513. 10.1042/BJ20101764 . hal-00608395

\section{HAL Id: hal-00608395 \\ https://hal.science/hal-00608395}

Submitted on 13 Jul 2011

HAL is a multi-disciplinary open access archive for the deposit and dissemination of scientific research documents, whether they are published or not. The documents may come from teaching and research institutions in France or abroad, or from public or private research centers.
L'archive ouverte pluridisciplinaire HAL, est destinée au dépôt et à la diffusion de documents scientifiques de niveau recherche, publiés ou non, émanant des établissements d'enseignement et de recherche français ou étrangers, des laboratoires publics ou privés. 


\section{Evidence that ACETATE NON-UTILIZING 1 prevents carbon leakage from peroxisomes during lipid mobilization in Arabidopsis seedlings ${ }^{1}$}

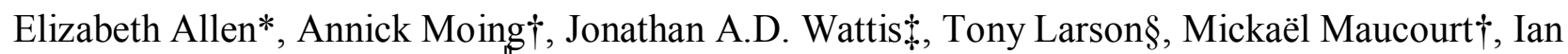
A. Graham§, Dominique Rolin \& Mark A. Hooks*

* School of Biological Sciences, Bangor University, Bangor, Gwynedd LL57 2UW, United Kingdom

† Plateforme Métabolome du Centre de Génomique Fonctionnelle Bordeaux, IFR103 BVI, BP 81, F-33140 Villenave d'Ornon, France

† School of Mathematical Sciences, University of Nottingham, University Park, Nottingham NG7 2RD, United Kingdom

$\S$ Centre for Novel Agricultural Products, University of York, Heslington, York YO10 5DD United Kingdom

"INRA, Université de Bordeaux, UMR619 Fruit Biology Unit, BP 81, F-33140 Villenave d'Ornon, France (DR)

Running head: Acetate Metabolism and Signaling

Corresponding author:

Mark A. Hooks

School of Biological Sciences

College of Natural Sciences

University of Wales

Bangor, Gwynedd LL57 2UW

United Kingdom

Phone: +441248382297

Fax: +441248370731

Email: m.a.hooks@bangor.ac.uk

Key words: acetyl-CoA, Arabidopsis, glyoxylate cycle, metabolism, modelling, substrate cycle 


\section{Synopsis}

ACETATE NON-UTILIZING 1 (ACN1) is a short-chain acyl-CoA synthetase, which activates acetate to acetyl-CoA in peroxisomes of Arabidopsis. Pulse-chase $\left[2-{ }^{13} \mathrm{C}\right]$ acetate feeding of the mutant acn 1-2 revealed that acetate accumulation and assimilation were no different to that of wild-type, Col-7. However, the lack of acn 1-2 led to a decrease of nearly $50 \%$ in ${ }^{13} \mathrm{C}$-labelling of glutamine, a major carbon sink in seedlings, and large decreases in primary metabolite levels. In contrast, acetyl-CoA levels were higher in acn1-2 compared to Col-7. The disappearance of eicosenoic acid was slightly delayed in acn1-2 indicating only a small effect on the rate of lipid breakdown. A comparison of transcript levels in acn1-2 and col-7 showed that induced genes included a number of metabolic genes and also a large number of signaling-related genes. Genes repressed in the mutant were represented primarily by embryogenesis related genes. Transcript levels of glyoxylate cycle genes also were lower in acn 1-2 than in Col-7. We conclude that deficiency in peroxisomal acetate assimilation comprises only a small proportion of total acetate use, but this affects both primary metabolism and gene expression. We discuss the possibility that ACN1 safeguards against the loss of carbon as acetate from peroxisomes during lipid mobilization.

\section{INTRODUCTION}

The process of seed germination has been delineated into three steps, water absorption by the seed coat, the initiation of metabolic processes within the outer cellular layers (the endosperm of oilseeds) and the establishment of metabolic processes within the embryo leading to radical emergence from the seed coat [1]. In the oilseed Arabidopsis, the cotyledons of the embryo contain about $90 \%$ of the total triacylglycerol (TAG) for carbon nutrition. The endosperm contains about $10 \%$ of the total seed TAG, degradation of which is essential to kick-start embryo metabolism and permit normal development of the seedling [2]. Prior to seedling emergence the degradation of TAG begins in earnest in order to feed the rapidly developing seedling and they are completely consumed by the time the cotyledons have spread and greened completely. Fatty acids released from TAGs are transported to glyoxysomes where they are activated to their acyl-CoA esters to enter $\beta$-oxidation [3]. The $\beta$-oxidation spiral releases acetyl-CoA, which enters the glyoxylate cycle for conversion to organic acids that can be exported to mitochondria for subsequent gluconeogenesis [4] or to fuel respiration [5, 6]. However, only recent work on Arabidopsis mutants has revealed the enzymes critical for linking $\beta$-oxidation to primary carbon metabolism. At least one glyoxysomal isozyme of citrate synthase and malate dehydrogenase are essential for lipid catabolism and seedling establishment, whereas isocitrate lyase and malate synthase are not [7].

The glyoxylate cycle and its association with $\beta$-oxidation were elucidated almost 50 years ago through feeding studies using radiolabelled acetate [8]. For exogenous acetate to be assimilated through the glyoxylate cycle, it requires transport into the peroxisome and activation to acetyl-CoA. Through the characterization of mutants resistant to fluoroacetate [9], we demonstrated that the ATP-binding cassette protein COMATOSE [10] and the short-chain acyl-CoA synthetase ACN1/AAE7 [11, 12], are the primary factors for acetate transport and activation, respectively. COMATOSE appears to transport a wide variety of fatty acid related compounds, thus the relative importance of acetate transport remains to be elucidated. The transport and activation of fatty acids by long-chain acyl-CoA synthetases is essential $[13,14]$, but a short-chain/acetyl-CoA enzyme activity would seem superfluous, since it resides outside the normal pathway of fatty acid degradation.

Although acetyl-CoA is a metabolite pivotal to central metabolism as a biosynthetic precursor, defining the roles that acetyl-CoA synthetases play in generating acetyl-CoA remains elusive [15]. Through their characterisation of the acetyl-CoA synthetase mutant acs1, Lin and Oliver [16] estimated that this enzyme is responsible for up to $90 \%$ of the total acetyl-CoA synthetase (ACS) activity in older seedlings. Accordingly, they postulated that ACS1 may be an 
important means by which acetate is recycled in Arabidopsis. However, ACS activity also resides within the cytosol, which is greater than either plastid or peroxisome associated activities [12]. Several objectives toward ascertaining the importance of ACN1/AAE7 in developing seedlings were to determine the effects of eliminating this step on metabolite labelling patterns, metabolite levels and gene expression profiles using the acn 1-2 mutant. We observed changes to the labelling of the sink metabolite Gln and in the levels of both metabolites and transcripts. This suggests an important metabolic role of ACN1/AAE7 during lipid mobilization, which may subsequently alter transcriptional responses. These results highlight the still unknown complexity of carbon utilization and partitioning and its regulation during seedling establishment. We discuss our data as a foundation of mathematical modelling efforts to explain the metabolic differences between acn1-2 and Col-7, which suggest a role of ACN1/AAE7 to prevent carbon loss from peroxisomes during lipid mobilization [17].

\section{EXPERIMENTAL}

\section{Plant material and growth conditions}

All seeds were surface sterilized and imbibed in the dark at $4^{\circ} \mathrm{C}$ for 3 days before sowing onto agar plates. For all experimental conditions, seeds were germinated at $20^{\circ} \mathrm{C}$ at $70 \mu \mathrm{mol}$ of photons $/ \mathrm{m}^{2} / \mathrm{s}$ constant illumination. Standard agar media plates contained $0.8 \%$ agar, $1 / 2$-strength MS salts [18]. The media, prior to addition of agar and subsequent autoclaving, was adjust to $\mathrm{pH}$ 5.7 with $0.1 \mathrm{~N} \mathrm{KOH}$. In order to obtain conclusive results regarding the behaviour of mutant compared to wild-type seedlings from the metabolite and transcript profiling, it was necessary to obtain equally developed seedlings. Due to the more rapid development of acn1-2 [9] (6 to $8 \mathrm{~h}$ before Col-7 to attain Principal Growth Stage (PGS) 0.7 [19]) we selected batches of seedlings with approximately the same average fresh weight in addition to visually staging seedling development. The average fresh weights of 50 seedlings from plates used for RNA extraction were $8.8 \pm 1.2$ $(\mathrm{n}=8)$ and $8.9 \pm 1.3(\mathrm{n}=8)$ for Col-7 and $a c n 1-2$, respectively. All seedling batches used for metabolite quantification and feeding studies were selected to fall within these weights.

\section{$\left[{ }^{13} \mathrm{C}\right]$ Acetate and $\left[{ }^{13} \mathrm{C}\right] \mathrm{GIn}$ quantification}

Seedlings were washed from standard agar media plates using distilled water, rinsed well, and $\sim 1 \mathrm{~g}$ of seedlings was bubbled with air in a sterile solution of 1/2-strength MS salts, $20 \mathrm{mM}$ sucrose, and $50 \mathrm{mM}$ MES, pH 5.7. After 1 hour, $\left[2-{ }^{13} \mathrm{C}\right]$-labelled sodium acetate was added to a concentration of $4 \mathrm{mM}$ and the seedlings incubated for designated lengths of time. Chase experiments were performed by transferring seedlings to an air-bubbled solution of $4 \mathrm{mM}$ unlabelled acetate two hours after adding the $\left[2-{ }^{13} \mathrm{C}\right]$ acetate, and continuing the incubations for either 2 or $4 \mathrm{~h}$. At each sampling time, the seedlings were removed from the solution, rinsed thoroughly with distilled $\mathrm{H}_{2} \mathrm{O}$, and frozen in liquid nitrogen. Metabolites were extracted according to Weckwerth et al. [20] and lyophilised overnight. The residue was resuspended in $550 \mu 1$ of an aqueous solution containing $18 \%(\mathrm{v} / \mathrm{v}) \mathrm{D}_{2} \mathrm{O}, 1.75 \mu \mathrm{g} / \mathrm{ml}$ streptomycin, $3.5 \mu \mathrm{g} / \mathrm{ml} \mathrm{NaN}$, and $8 \mathrm{mM}$ ${ }^{13} \mathrm{C}$-formate, which served as an internal standard. The $\mathrm{pH}$ was not adjusted. Standard broadband, proton-decoupled, Fourier transform NMR ${ }^{13} \mathrm{C}$ spectra were acquired on a Bruker AC250 spectrometer operating at $62.9 \mathrm{MHz}$ The interval between $90^{\circ}$ pulses was $2 \mathrm{~s}$, and the spectral width and data points were $16000 \mathrm{~Hz}$ and $8 \mathrm{~K}$, respectively.

\section{Metabolite quantification}

Approximately $0.6 \mathrm{~g}$ of seedlings was washed from the surface of the agar petri dishes with distilled sterile water into a filtration unit. Once the water had passed through the seedlings were washed in $10 \mathrm{ml}$ more water, weighed and immediately frozen in liquid nitrogen. The time from opening the petri dish to freezing the sample was approximately $4.5 \mathrm{~min}$. Metabolites were 
extracted according to Weckwerth et al. [20] and metabolite quantification by ${ }^{1} \mathrm{H}-\mathrm{NMR}$ was conducted as described in Moing et al. [21]. Briefly, the dried extracts were resuspended in 400 $\mathrm{mM}$ phosphate buffer $\mathrm{pH} 6.0$ in $\mathrm{D}_{2} \mathrm{O}$ and analyzed at $500.162 \mathrm{MHz}$ on a Bruker spectrometer (Bruker Biospin Avance). Special care was taken to allow absolute quantification of the individual metabolites through addition of ethylene diamine tetraacetic acid sodium salt solution ( $5 \mathrm{mM}$ final concentration in the NMR tube) to improve the resolution and quantification of organic acids such as malate and citrate, adequate choice of the NMR acquisition parameters (pulse angle $90^{\circ}$, relaxation delay $10 \mathrm{~s}$ ) and use of an electronic reference calibrated with glucose, fructose, Gln and Glu sodium salt solutions as described previously [21]. Individual metabolites were identified using published data [21], acquisition of NMR spectra of reference compounds under exact solvent conditions, and spiking extracts with reference compounds. They were quantified using the metabolite mode of AMIX software (Bruker Biospin v. 3.5.6) based on the number of protons comprising the corresponding resonance.

Samples for fatty acid measurements were sown onto agar plates and tissue samples harvested after the same length of time under the growth conditions specified above. Samples of 50 seeds or 25 seedlings were collected by forceps, weighed in screw-cap tubes, and immediately frozen in liquid nitrogen. Fatty acids were measured using the method of Browse et al. [22]. AcetylCoA levels were determined by HPLC according to Larson et al. [23]. Acetyl-CoA extraction efficiency and relative quantification was confirmed using the extraction protocol of Tumaney et al. [24].

\section{Transcript profiling and data analysis}

Total RNA was extracted from approximately $0.5 \mathrm{~g}$ of PGS 0.7 seedlings. The RNA was sent to the GARNet transcript profiling service at Nottingham University for quality analysis, dye labelling and hybridisation to the Affymetrix ATH1 GeneChip ${ }^{\mathrm{TM}}$ arrays. Twelve arrays were produced with one target hybridised to each chip. Three replicates of two sample types were prepared of Col-7 and acn1-2. The data pre-processing was carried out at the GARNet transcript profiling service where poor hybridisation events were noted and quantification of hybridisation intensity was performed. Further data mining was carried out at UWB using GeneSight ${ }^{\mathrm{TM}} \mathrm{v}^{4} .1$ (Biodiscovery Inc., San Mateo, USA). The raw expression profiling data is available from the GARNet transcript profiling service (affymetrix.arabidopsis.info). Microarray expression data and all associated experimental metadata were presented to comply with MIAME guidelines [25].

Identification of differentially expressed genes between acn1-2 and Col-7 was conducted using GeneSight ${ }^{\mathrm{TM}}$ v 4.1 (BioDiscovery Inc.). Unless stated otherwise, data normalisation was performed using the pre-set "Affy" data transformation sequence. A dataset was created in GeneSight in order to identify gene expression ratios between Col-7 and acn1-2 that were consistent throughout the microarrays. First, three groups were created that gave gene expression ratios for each acn 1-2 replicate compared to one of the three Col-7 replicates. For example, group 1 comprised expression ratios calculated for every pair between acn1-2 (1) and Col-7 (1), acn1-2 (2) and Col-7 (1), and acn 1-2 (3) and Col-7 (1). To do this, signal intensities in this dataset were transformed, except that replicates were not combined as the final step of the pre-set 'Affy' transformation process. This kept the data within two categories each containing the minimum three replicates needed in order to conduct the ANOVA $(\mathrm{P}<0.05)$ to establish the set of consistent ratios between acn 1-2 and Col-7 (GeneSight Users Manual v. 4.1, 2003). This set of 14,256 genes represented the core set of genes common to each group from which differentially expressed genes were determined.

We adopted a combined approach of using a discretionary 'fold' cut-off level in conjunction with further statistical tests to determine the group of DE genes. A value at which genes showed DE was first identified based on reiterative hierarchical clustering of genes at various fold-change thresholds. The determined fold cut-off level was used in conjunction with a t-test $(P<0.05)$ to 
identify a statistically confident group of genes that showed DE between acn1-2 and Col-7. For each group, the $\log _{2}$ differences in gene expression of the 14,256 genes were calculated between acn1-2 and Col-7 replicate. DE genes were identified in each group using a bootstrapping algorithm [26]. Through a reiterative process of decreasing the fold cut-off value while maintaining $95 \%$ confidence limits we obtained a set of 201 genes showing DE between acn 1-2 and Col-7 at a cut-off threshold of 1.4-fold. Twenty of twenty times, hierarchal clustering of this set of genes resulted in complete separation of acn 1-2 from Col-7. The hierarchical clustering was employed to determine the optimal number of windows to create 2-D self-organising maps to explore the structure of the data.

\section{RESULTS}

\section{Global acetate consumption appears normal}

We had previously reported that the acn 1-2 mutant appeared to be less tolerant of acetate during the prolonged exposure provided through germination and emergence on standard agar media plates containing acetate [9]. We hypothesized that the lack of acetate assimilation resulted in accumulation that would lead to acidification of the cytosol and ultimately death of the seedlings. Accordingly, the relatively high tolerance of the mutant to butyrate compared to acetate reflected the greater importance of ACN1 in activating acetate, and that butyrate may be activated by another enzyme. In previous $\left[2-{ }^{14} \mathrm{C}\right]$ acetate feeding studies on similarly staged seedlings, substantial label appeared in non-carbohydrate compound classes at near wild-type levels, except for the ethanol insoluble and organic acid fractions [12]. This indicated that acetate metabolism was not completely abolished, but it was unclear how much it was reduced in the mutant. The relatively higher level of label in the organic acid fraction seen in the mutant from that study may have come from higher levels of un-metabolised free acetate indicating reduced assimilation. In order to test this hypothesis, we compared the uptake and utilization of acetate by acn1-2 compared to Col-7. Using NMR, we quantified the resonance corresponding to the $\mathrm{C} 2$ carbon of $\left[2-{ }^{13} \mathrm{C}\right]$ acetate-fed seedlings. It was necessary to conduct the feeding experiment in the presence of sucrose for observable levels of acetate to be taken up. Not only was sucrose promoting uptake, it was also exerting a protective effect on the seedlings. Seedlings fed acetate alone had a barely detectable ${ }^{13} \mathrm{C}$ signature after even $8 \mathrm{~h}$, and seedlings rapidly deteriorated as observed by their tendency to fall apart during harvest. This was not unexpected as the toxic nature of free acetate on Arabidopsis seedlings and plant cells had already been reported [27]. The degree of deterioration at any given time was comparable between mutant and Col-7 during incubation times up to $24 \mathrm{~h}$. Having sucrose present permitted us to extract and estimate free acetate levels for up to $24 \mathrm{~h}$ after feeding started. There was no difference in labelled acetate levels between acn1-2 and Col-7 (Fig. 1). Isotopically labelled acetate increased from undetectable to maximum levels of $40-50 \mu \mathrm{mol} \mathrm{g} \mathrm{g}^{-1}$ fwt within $2 \mathrm{~h}$. Levels remained elevated for $24 \mathrm{~h}$ with Col-7 and acn 1-2 having $29 \pm 11$ and $41 \pm 18 \mu \mathrm{mol} \mathrm{g}^{-1} \mathrm{fwt}$, respectively. This shows that labelling of the endogenous acetate pool reaches steady-state within $2 \mathrm{~h}$, and that we were measuring the capacity of the entire seedling to take up acetate and not just a specific tissue. To test if acetate was being metabolised, samples were chased with isotopically normal acetate and the fate of labelled acetate followed. Acetate had decreased within two hours to about $10 \mu \mathrm{mol} \mathrm{g}{ }^{-1}$ fwt. The fall in labelled acetate was similar for both acn1-2 and Col-7. In the subsequent $2 \mathrm{~h}$, only a small further decrease was observed.

\section{Disruption of the flow of carbon from acetate into Gin}

Under the nitrogen replete conditions that we used, the predominant sink for label was Gln, and it appeared in all three carbons C2, C3 and C4 in the NMR spectra. We determined the levels of Gln based on each of the three labelled carbon atoms in the same extracts used for acetate quantification in Col-7 and acn1-2 (Figs. 2A-C). In both Col-7 and acn1-2, label in C2 appeared to 
reach steady-state within $2 \mathrm{~h}$. In Col-7, the amount of label in C3 and C4 continued to increase up to $4 \mathrm{~h}$ after feeding, but reached steady-state levels in acnl-2 by $2 \mathrm{~h}$. At $4 \mathrm{~h}$, labelling in $\mathrm{C} 3$ and $\mathrm{C} 4$ were maximal in Col-7 (data not shown). By $4 \mathrm{~h}$, there was approximately a 50\% reduction in the amount of label appearing in the $\mathrm{C} 3$ and $\mathrm{C} 4$ carbons in acn 1-2. There was only a slight reduction in label at position $\mathrm{C} 2$ for acn1-2. The differences in the amount of total label in each carbon and the timing in reaching apparent steady state, suggested that the Gln pool size may be smaller in acn 1-2 than in Col-7. Upon chasing with isotopically normal acetate, the levels of label in Gln remained level or rose in the wild-type over the following $2 \mathrm{~h}$. However, for acn 1-2, there was no change or a slight decrease in labelled Gln post-chase, which showed that the flow of label from acetate to Gln was affected.

\section{Depression of major metabolite levels in acn1-2}

The timing and degree of Gln labelling suggested that the pool size was less in acn1-2 than in Col-7. To confirm our conclusion, the levels of Gln and other metabolites were quantified by ${ }^{1} \mathrm{H}$ NMR. We quantified 27 distinct resonances corresponding to a variety of known and unknown metabolites, including soluble carbohydrates, amino acids and organic acids (Table I). Acetyl-CoA levels were determined by HPLC [23]. In general, the absolute levels of amino acids quantified on a $\mathrm{nmol} \mathrm{g}^{-1}$ fwt basis were of the same order as those observed in seeds of the Arabidopsis ecotype Wassilewskija [28] or in seed germination series for other species, such as the legumes soybean and lupin [29].

On average, Gln was 70\% lower in acn 1-2 than in Col-7, which corresponds to the labelling data. Furthermore, there was a depression of primary metabolite levels in acn1-2, which extended to all classes of metabolites that were NMR visible and quantifiable (Table I). The change was significant at $p<0.05$ for every compound measured except fumarate. Individual amino acids in acn $1-2$ were lower by $67 \%$, on average, to those in Col-7, and ranged from $80 \%$ less proline to $59 \%$ less leucine. Individual soluble sugars in acn1-2 were on average $63 \%$ lower than those in Col-7 with the largest decrease observed for rhamnose at $69 \%$. When fumarate was excluded from the calculation, organic acids in acn1-2 were on average $58 \%$ lower to the levels of corresponding ones in Col-7, ranging from $70 \%$ less formate to $40 \%$ less citrate. Malate was $50 \%$ lower in acn $1-2$. The unknown compounds were affected similarly to the other classes with levels between 40 and $60 \%$ lower. Fumarate showed a small, but insignificant increase in the mutant. If one effect of the mutation is to reduce carbon flow through the glyoxylate cycle via isocitrate lyase, then a potential decrease in succinate levels could have a corresponding effect on fumarate levels. If succinate was not the primary organic acid transported into mitochondria, since ICL activity appears unnecessary in light grown seedlings [6], then fumarate may be a metabolite not affected by the anapleurotic/gluconeogenic functions of glyoxylate/TCA cycle couple. In contrast to the behaviour of primary metabolites, acetyl-CoA was found to be more than 2-fold greater in acn1-2 than in Col7.

\section{Measurements of fatty acid catabolism}

One possibility for lower metabolite levels in acn1-2 may have been due to less carbon entering metabolism from lipid mobilization. We conducted a time-course analysis of the disappearance of eicosenoic acid (C20:1), which is a marker of TAG degradation [30], at three different time points, imbibed seeds and 3 and 5 days post-imbibition (Fig 3). The amount of eicosenoic acid in seeds was similar suggesting that fatty acid and TAG synthesis and storage is not affected in developing seeds. During germination and seedling growth, virtually all eicosenoic acid was gone in both Col-7 and acn1-2 by day 5. For both genotypes, the decrease in eicosenoic acid was significant between successive time points and the difference in levels between acn1-2 and Col-7 was significant at day 3 , despite the seedlings being at a slightly more advanced stage of growth. 


\section{Transcript profiling}

A group of differentially expressed genes were identified in each dataset using an algorithm based on the bootstrapping algorithm developed by Kerr and Churchill [26]. Genes were assigned as being either differentially expressed or not, based on the intensity of the replicated spots. For a gene to be identified as differentially expressed between acn1-2 and Col-7, the gene expression values from all three replicated microarrays need to be identified as differentially expressed. We employed a reiterative process of decreasing the fold cut-off value while maintaining $95 \%$ confidence limits [26] for DE among replicates. In this manner we obtained a set of 201 genes showing DE between acn1-2 and Col-7 at a cut-off threshold of 1.4-fold, which represented a statistically confident group of DE genes. Hierarchal clustering of the DE genes showed consistent segregation of seedling samples between mutant and Col-7 (Supplemental Fig. 1) and that the optimal number of bins for visualisation of the data by 2D-SOM was 9 (Supplemental Fig. 2). Three (bins 7-9) and six (bins 1-6) of the nine bins contained genes that were up- and downregulated, respectively (Supplemental Fig. 3). Of the 201 DE genes, 71 were up-regulated in acn 1-2 with an increase in expression from 1.8 to 27.7 -fold, whereas 130 genes were down-regulated with a decrease in expression ranging from 3.8 to 52.87 -fold. All the DE genes are listed according to bin number for which repetitive mapping gave the greatest proportion of instances a gene fell into a particular bin (Supplemental Table 1).

The up- and down-regulated sets of genes were analysed for ontological patterns according to the bins assigned by the 2D-SOM [31]. A variety of genes with assigned identity were found in each cluster. Of the genes with assigned biological function based on ontology, Cluster 1 was comprised of genes involved in developmental processes, protein metabolism, nucleic acid related and signal transduction. Cluster 2 contained genes for protein metabolism, signalling and transcription. Cluster 3 contained genes involved in responses to stress or external stimuli, development, signalling and transcription. Genes from clusters 4 and 5 were examined jointly as these genes clustered together intermittently upon repeated 2D-SOM clustering, but no enrichment of a particular type of gene was noted. Similarly, genes in cluster 6 did not show a bias towards any specific function or process. Analysis of cluster 7 showed that the majority of genes were involved in response to stress or external stimuli, signalling and nucleic acid related. The genes of cluster 8 did not show enrichment in any specific ontology. Analysis of cluster 9 showed the genes were mainly involved in transcription or development.

We examined more closely the top twenty up- and down-regulated genes (Table 2) following the assumption that the genes that demonstrate the largest change in expression levels between two samples are the pivotal genes around which the difference in biological basis revolves [32]. Of the twenty genes that showed the greatest increase in expression, 16 had a predicted biological function. These included a gene associated with the phytohormone auxin was induced (At5g13350) as well as a number of genes possibly involved in the regulation of translation or transcription (At3g49460, At1g49920, At5g19920, At2g46780, and At2g17920). Examining the top twenty down-regulated genes in acn1-2, the identities of 16 were assignable. Eight of which were involved with early seedling development, including genes encoding late embryogenesis abundant (LEA) proteins (At2g36640, At1g32560, At4g36600, At4g21020), seed storage protein (At5g39110) and a protein associated with embryonic development (At5g62210). Genes associated with hormone regulators of plant development At1g48660 (auxin) and At3g02480 (ABA) and a Ring-finger family transcription factor (At4g35480) were also repressed in acn1-2.

\section{Transcript levels of genes associated with the assimilation of acetate}

Since the sequential action of the glyoxylate cycle, TCA cycle and gluconeogenesis is important for assimilating both free acetate and acetyl-CoA from $\beta$-oxidation, it was of particular interest to examine what happened with the expression of glyoxylate cycle, anapleurotic and 
gluconeogenic genes in acn1-2. None of the genes examined showed differential expression between acn 1-2 and Col-7 when the 1.4-fold change in gene expression threshold was used in conjunction with a t-test $(P>0.05)$. However, certain genes showed DE when a simple threshold cut-off value of +/- 1.4-fold was applied (Table 2). Two genes, a putative cytosolic malate dehydrogenase (At5g56720) and one phosphoenolpyruvate carboxykinase (PCK) showed an increase in transcript levels in acn1-2 of 2.9- and 2.0- fold, respectively. The putative PCK is not the one known to be developmentally important during early seedling establishment [30]. The genes for which transcript levels were lower in acn1-2 were malate synthase (At5g03860) [33], isocitrate lyase (At3g21720) [6], a malate dehydrogenase-related protein (At3g53910), citrate synthase 3 (CSY3, At2g42790) [34], an aspartate aminotransferase (ASP1, At2g30970) [35] and ACN1/AAE7 (At3g16910) [12] in the range of 5.5- to 1.4- fold. We have shown previously that a partial transcript for ACN1/AAE7 is detectable in the acn1-2 mutant [12], therefore, its expression in the mutant could be determined.

\section{DISCUSSION}

We have employed a combination of metabolite and transcript profiling to investigate the effects on metabolism and gene expression caused by eliminating the peroxisomal acetyl-CoA synthetase step catalysed by ACN1/AAE7. Because other acetyl-CoA synthetase activities are present in Arabidopsis [12, 36], it was necessary to determine if the lack of ACN1/AAE7 affected the overall assimilation of acetate. It was evident from our previous studies that acetate metabolism is not greatly compromised in acn1-2. However, this component of acetate assimilation represents the gluconeogenic contribution as shown by the large decrease in $\left[{ }^{14} \mathrm{C}\right]$ acetate appearing in soluble carbohydrate in the acn1-2 and comatose/acn 2 mutants compared to wild-type [10, 12]. We conclude that the effects that we observe on metabolite levels and gene expression reflect a defect in a relatively narrow, but metabolically important, aspect of acetate assimilation.

One possibility is that it serves to recycle acetate in young seedlings. Several mechanisms for acetate production have been described [37]. For example, cysteine biosynthesis is likely to be a substantial producer of free acetate. In loblolly pine, free cysteine levels go from being essentially undetectable in dry seeds, in contrast to all other amino acids, to become the tenth most abundant free amino acid within several days of radical emergence [38]. Furthermore, the flux through cysteine would be high, since it serves as the route by which sulphur is assimilated into proteins and metabolic intermediates [39] and a variety of sulphur-containing defence compounds [40]. Therefore, it is highly likely that cysteine biosynthesis with contributions from protein deacetylation provides a steady and high flux of free acetate within developing seedlings. In our study, the amount of labelled acetate did not change dramatically during the second two hours of the chase, thereby suggesting that it continues to be produced. We have shown that plant cells have an enormous capacity to accumulate acetate if presented with an exogenous source, but that levels of free acetate in untreated seedlings are very low. Thus, it appears that Arabidopsis seedlings have a large capacity to turn-over free acetate. From the disappearance of label in acetate, the turnover of the acetate pool is estimated to be about $5.4 \mathrm{nmol} \mathrm{s}^{-1} \mathrm{~g}^{-1}$ fwt and the entire acetate pool could turn over in approximately $8 \mathrm{~s}$. It must be noted that this rate is an estimate from sucrose-fed seedlings and the rate may be different in non-treated seedlings. However, we previously reported that most free acetate assimilation is ACN1 independent in seedlings not fed with sucrose, and a large cytosolic ACS activity in seedlings that may account for acetate activation [12]. Therefore, we can conclude that re-assimilation of extra-peroxisomal acetate is not likely to be a major function of ACN1 in young seedlings. This function would not appear to fall onto ACS either, since acs 1 does not appear to be affected by exogenous acetate in seedlings less than 10 days old [16]. 
The key difference between mutant and wild-type that suggests an intra-peroxisomal function in acetate cycling is the difference in acetyl-CoA levels. Greater acetyl-CoA levels in acn1-2 appear counterintuitive for an enzyme that would convert acetate to acetyl-CoA. However, these results are consistent with a model of lipid mobilization where ACN1/AAE7 prevents the loss of carbon from peroxisomes as free acetate by reactivating it (Fig. 4). Acetyl-CoA cannot cross the peroxisomal membrane [41], but there are a number of acyl-CoA thioesterases present in peroxisomes of Arabidopsis, some of which are active with short-chain substrates or the substrate specificity is currently unknown [42, 43]. This situation would be analogous to the increase in acetyl-CoA levels observed for the heterologous expression of the $S$. cerevisiae acetyl-CoA hydrolase in mitochondria of tobacco plants [44]. Their explanation was that free acetate is exported from mitochondria and reactivated in the cytosol. The lack of acetate reactivation in peroxisomes would not expect to have the severe consequences on plant growth as observed for the acetyl-CoA hydrolase expressing plants, because sufficient acetyl-CoA would be produced by $\beta$-oxidation. Increased levels of cytosolic acetyl-CoA may even promote biosynthetic processes allowing faster development under ideal growing conditions. A similar conclusion was made about an aconitase isozyme of tomato that was hypothesized to be a factor affecting the distribution of carbon between the TCA cycle and sucrose biosynthesis [45]. Elimination of this aconitase resulted in reduce levels of primary metabolites and an increase in various soluble sugars indicating a shift in carbon allocation from respiration to biosynthetic processes. The tomato aconitase mutant grew taller and produced greater fruit mass than the corresponding wild-type plant. The lack of ACN1/AAE7 may affect the rate at which acetyl-CoA enters the glyoxylate cycle from fatty acid degradation. The opposing activities of ACN1/AAE7 and a thioesterase, in conjunction with the ability of peroxisomes to import and export acetate, would constitute a true substrate cycle [46]. Metabolic rather than transcriptional control of lipid mobilization processes may explain why the expression of $\beta$-oxidation and glyoxylate cycle genes is unaffected even in situations where lipid mobilization is severely diminished [34], and why we observed an effect on the expression of very few metabolic genes. It is known that germination and lipid mobilization are regulated independently [47]. Whereas the molecular mechanisms of germination are quite well defined, the regulation of metabolic processes remains poorly understood.

We conducted mathematic modelling to further explore the possibility that ACN1/AAE7 is necessary to prevent acetate leakage from peroxisomes [17]. The aim was to model the situation during lipid mobilization with acn1-2 containing higher levels of acetyl-CoA than Col-7. The assumptions placed into the model were: i) a steady-state production of acetyl-CoA from lipid mobilization, which is likely to be the case just after seedling emergence [48], ii) a higher rate of acetyl-CoA production from fatty acid degradation than from extra-peroxisomal sources, iii) an increase in ACN1 activity during seedling growth, iii) an extra-peroxisomal source of acetyl-CoA activity and a peroxisomal thioesterase active with acetyl-CoA, iv) the pool of primary metabolites is fed by fatty acid degradation via steps of the glyoxylate cycle, v) acetyl-CoA can be derived from the metabolite pool. Through extensive varying of parameters to mimic various conditions of metabolic fluxes, we could not obtain the observed differences in acetyl-CoA levels by forming acetyl-CoA exclusively via input from the metabolite pool. In addition, it would not seem likely that a reduced metabolite pool would generate higher acetyl-CoA levels in the mutant. However, we could produce models fulfilling these observations by having acetate leave peroxisomes and activated by an external acetyl-CoA synthetase as long as this activity was greater than that of ACN1/AAE7. More extensive modelling of acn1-2 metabolism using with targeted label input, such as through labelled fatty acids, at different developmental stages will be required to solidify our conclusions.

From gene expression profiles taken over the course of seedling establishment we have evidence that seedlings gradually attain photosynthetic competence after germination, and it is not complete until the first true leaves begin to appear [49]. This suggests that transcriptional programs 
promoting development are moderated by some mechanism, which may involve catabolite repression [50] and/or genetic factors. It has been hypothesized that genetic factors present in the embryo to regulate cotyledon expansion may continue to exert their effects well into establishment [51]. Our results show that both development and gene expression are affected by elimination of ACN1/AAE7. When global patterns of gene expression in acn1-2 seedlings are compared to Col-7 at the same stage of development, we see lower transcript levels of genes that are associated with embryogenesis in acn 1-2. Subsequently, the down-regulation of genes, such as the LEA proteins, may promote seedling growth like we observe with acn1-2. Genetic mechanisms preventing unimpeded (or causing suboptimal) growth have been demonstrated for the microorganism $B$, subtilis [52]. In general, transcript levels of acetate assimilation genes are also lower in $a c n 1-2$. This may reflect the lower rate at which fatty acids appear to be degraded in acn1-2 or could be the result of a diminished metabolic signal from a reduced primary metabolite pool. At this time it is not possible to conclude if altered gene expression is a cause or result of perturbed development, but it is evident that metabolic processes involving peroxisomal acetate/acetyl-CoA metabolism are important for normal seedling development. It is likely that ACN1/AAE7 begins to exert it influence on peroxisomal metabolism during imbibition. It is expressed in imbibed seeds and fluoroacetate, which is activated by ACN1/AAE7, greatly delays germination when seeds are imbibed in the presence of it [12]. In conclusion, our results support the findings of Bender-Machado et al. [44] and Oliver et al. [15] that acetate and acetyl-CoA levels are tightly regulated, and disrupting peroxisomal acetyl-CoA metabolism during germination affects both metabolism and gene expression and appears to have knock-on consequences to germination and seedling development.

\section{Acknowledgements}

We would like to thank Dr. Martine Dieuaide at INRA-Bordeaux for helping with the ${ }^{13} \mathrm{C}$ NMR, the GARNet microarray facility at the University of Nottingham for carrying out the array hybridization, and Professor A. Deri Tomos for aiding in the supervision of Elizabeth Allen. We would also like to acknowledge the contribution of the following people to the modelling effort: Martin Berglund, Hilmar Castro, Anna Lovrics, Tal Pearson, Nikolas Popovich, Jamie Twycross, Maria Grazia Vigliotti and Minaya Villasania. The PhD studentship funding for Elizabeth Allen came from the Sir Williams Roberts trust provided to Bangor University. The work was funded by the UK Biotechnology and Biological Sciences Research Council [grant no. P19408]. 


\section{References}

1 Bewley, D. J. and Black, M. (1994) Seeds: Physiology of Development and Germination. Plenum, New York

2 Penfield, S., Rylott, E. L., Gilday, A. D., Graham, S., Larson, T. R. and Graham, I. A. (2004) Reserve mobilization in the Arabidopsis endosperm fuels hypocotyl elongation in the dark, is independent of abscisic acid, and requires PHOSPHOENOLPYRUVATE CARBOXYKINASE1. Plant Cell. 16, 2705-2718

3 Graham, I. A. (2008) Seed storage oil mobilization. Annual Review of Plant Biology. 59, $115-142$

4 Beevers, H. (1961) Metabolic production of sucrose from fat. Nature. 191, 433-436

5 Raymond, P., Spiteri, A., Dieuaide, M., Gerhardt, B. and Pradet, A. (1992) Peroxisomal Beta-Oxidation of Fatty-Acids and Citrate Formation by a Particulate Fraction from Early Germinating Sunflower Seeds. Plant Physiology and Biochemistry. 30, 153-161

6 Eastmond, P. J., Germain, V., Lange, P. R., Bryce, J. H., Smith, S. M. and Graham, I. A. (2000) Postgerminative growth and lipid catabolism in oilseeds lacking the glyoxylate cycle. Proceedings of the National Academy of Sciences of the United States of America. 97, 5669-5674

7 Pracharoenwattana, I. and Smith, S. M. (2008) When is a peroxisome not a peroxisome? Trends in Plant Science. 13, 522-525

8 Canvin, D. T. and Beevers, H. (1961) Sucrose synthesis from acetate in the germinating castor bean: kinetics and pathway. The Journal of biological chemistry. 236, 988-995

9 Hooks, M. A., Turner, J. E., Murphy, E. C. and Graham, I. A. (2004) Acetate non-utilizing mutants of Arabidopsis: evidence that organic acids influence carbohydrate perception in germinating seedlings. Mol Genet Genomics. 271, 249-256

10 Hooks, M. A., Turner, J. E., Murphy, E. C., Johnston, K. A., Burr, S. and Jaroslawski, S. (2007) The Arabidopsis ALDP protein homologue COMATOSE is instrumental in peroxisomal acetate metabolism. The Biochemical journal. 406, 399-406

11 Shockey, J. M., Fulda, M. S. and Browse, J. (2003) Arabidopsis contains a large superfamily of acyl-activating enzymes. Phylogenetic and biochemical analysis reveals a new class of acylcoenzyme A synthetases. Plant Physiology. 132, 1065-1076

12 Turner, J. E., Greville, K., Murphy, E. C. and Hooks, M. A. (2005) Characterization of Arabidopsis fluoroacetate-resistant mutants reveals the principal mechanism of acetate activation for entry into the glyoxylate cycle. The Journal of biological chemistry. 280, 2780-2787

13 Fulda, M., Schnurr, J., Abbadi, A., Heinz, E. and Browse, J. (2004) Peroxisomal Acyl-CoA synthetase activity is essential for seedling development in Arabidopsis thaliana. Plant Cell. 16, 394-405

14 Baker, A., Graham, I. A., Holdsworth, M., Smith, S. M. and Theodoulou, F. L. (2006) Chewing the fat: beta-oxidation in signalling and development. Trends in Plant Science. 11, 124132

15 Oliver, D. J., Nikolau, B. J. and Wurtele, E. S. (2009) Acetyl-CoA-Life at the metabolic nexus. Plant Science. 176, 597-601

16 Lin, M. and Oliver, D. J. (2008) The role of acetyl-coenzyme a synthetase in Arabidopsis. Plant Physiology. 147, 1822-1829

17 Hooks, M. A., Allen, E. and Wattis, J. A. D. (2010) Modelling the peroxisomal carbon leak during lipid mobilization in Arabidopsis. Biochemical Society Transactions. 38, 1230-1233

18 Murashige, T. and Skoog, F. (1962) A revised medium for rapid growth and bioassay with tobacco tissue cultures. Physiologia Plantarum. 15, 473-496

19 Boyes, D. C., Zayed, A. M., Ascenzi, R., McCaskill, A. J., Hoffman, N. E., Davis, K. R. and Gorlach, J. (2001) Growth stage-based phenotypic analysis of Arabidopsis: a model for high throughput functional genomics in plants. Plant Cell. 13, 1499-1510 
20 Weckwerth, W., Wenzel, K. and Fiehn, O. (2004) Process for the integrated extraction identification, and quantification of metabolites, proteins and RNA to reveal their co-regulation in biochemical networks. Proteomics. 4, 78-83

21 Moing, A., Maucourt, M., Renaud, C., Gaudillere, M., Brouquisse, R., Lebouteiller, B., Gousset-Dupont, A., Vidal, J., Granot, D., Denoyes-Rothan, B., Lerceteau-Kohler, E. and Rolin, D. (2004) Quantitative metabolic pro. ling by 1-dimensional H-1-NMR analyses: application to plant genetics and functional genomics. Functional Plant Biology. 31, 889-902

22 Browse, J., McCourt, P. J. and Somerville, C. R. (1986) Fatty acid composition of leaf lipids determined after combined digestion and fatty acid methyl ester formation from fresh tissue. Anal Biochem. 152, 141-145

23 Larson, T. R., Edgell, T., Byrne, J., Dehesh, K. and Graham, I. A. (2002) Acyl CoA profiles of transgenic plants that accumulate medium-chain fatty acids indicate inefficient storage lipid synthesis in developing oilseeds. Plant Journal. 32, 519-527

24 Tumaney, A. W., Ohlrogge, J. B. and Pollard, M. (2004) Acetyl coenzyme A concentrations in plant tissues. J Plant Physiol. 161, 485-488

25 Brazma, A., Hingamp, P., Quackenbush, J., Sherlock, G., Spellman, P., Stoeckert, C., Aach, J., Ansorge, W., Ball, C. A., Causton, H. C., Gaasterland, T., Glenisson, P., Holstege, F. C., Kim, I. F., Markowitz, V., Matese, J. C., Parkinson, H., Robinson, A., Sarkans, U., Schulze-Kremer, S., Stewart, J., Taylor, R., Vilo, J. and Vingron, M. (2001) Minimum information about a microarray experiment (MIAME)-toward standards for microarray data. Nat Genet. 29, 365-371

26 Kerr, M. K. and Churchill, G. A. (2001) Bootstrapping cluster analysis: Assessing the reliability of conclusions from microarray experiments. Proceedings of the National Academy of Sciences of the United States of America. 98, 8961-8965

27 Graham, I. A., Leaver, C. J. and Smith, S. M. (1992) Induction of Malate Synthase Gene Expression in Senescent and Detached Organs of Cucumber. Plant Cell. 4, 349-357

28 Li, L., Thipyapong, P., Breeden, D. C. and Steffens, J. C. (2003) Overexpression of a bacterial branched-chain-keto acid dehydrogenase complex in Arabidopsis results in accumulation of branched-chain acyl-CoAs and alteration of free amino acid composition in seeds. Plant Science. 165, 1213-1219

29 Martínez-Villaluenga, C., Kuo, Y.-H., Lambein, F., Frías, J. and Vidal-Valverde, C. (2006) Kinetics of free protein amino acids, free non-protein amino acids and trigonelline in soybean (Glycine max L.) and lupin (Lupinus angustifolius L.) sprouts. Eur. Food Res. Technol. 224, 177186

30 Rylott, E. L., Gilday, A. D. and Graham, I. A. (2003) The gluconeogenic enzyme phosphoenolpyruvate carboxykinase in Arabidopsis is essential for seedling establishment. Plant Physiology. 131, 1834-1842

31 Berardini, T. Z., Mundodi, S., Reiser, L., Huala, E., Garcia-Hernandez, M., Zhang, P., Mueller, L. A., Yoon, J., Doyle, A., Lander, G., Moseyko, N., Yoo, D., Xu, I., Zoeckler, B., Montoya, M., Miller, N., Weems, D. and Rhee, S. Y. (2004) Functional annotation of the Arabidopsis genome using controlled vocabularies. Plant Physiol. 135, 745-755

32 Breitling, R., Armengaud, P., Amtmann, A. and Herzyk, P. (2004) Rank products: a simple, yet powerful, new method to detect differentially regulated genes in replicated microarray experiments. FEBS Lett. 573, 83-92

33 Cornah, J. E., Germain, V., Ward, J. L., Beale, M. H. and Smith, S. M. (2004) Lipid utilization, gluconeogenesis, and seedling growth in Arabidopsis mutants lacking the glyoxylate cycle enzyme malate synthase. Journal of Biological Chemistry. 279, 42916-42923

34 Pracharoenwattana, I., Cornah, J. E. and Smith, S. M. (2005) Arabidopsis peroxisomal citrate synthase is required for fatty acid respiration and seed germination. Plant Cell. 17, 20372048 
35 de la Torre, F., De Santis, L., Suarez, M. F., Crespillo, R. and Canovas, F. M. (2006) Identification and functional analysis of a prokaryotic-type aspartate aminotransferase: implications for plant amino acid metabolism. Plant J. 46, 414-425

36 Ke, J., Behal, R. H., Back, S. L., Nikolau, B. J., Wurtele, E. S. and Oliver, D. J. (2000) The role of pyruvate dehydrogenase and acetyl-coenzyme A synthetase in fatty acid synthesis in developing Arabidopsis seeds. Plant Physiol. 123, 497-508

37 Bao, X., Focke, M., Pollard, M. and Ohlrogge, J. (2000) Understanding in vivo carbon precursor supply for fatty acid synthesis in leaf tissue. Plant J. 22, 39-50

38 King, J. E. and Gifford, D. J. (1997) Amino Acid Utilization in Seeds of Loblolly Pine during Germination and Early Seedling Growth (I. Arginine and Arginase Activity). Plant Physiol. 113, $1125-1135$

39 Giovanelli, J., Mudd, S. H. and Datko, A. H. (1980) Sulfur amino acids in plants. In The Biochemistry of Plants (Miflin, B. J., ed.). pp. 375-402, Academic Press, New York

40 Rausch, T. and Wachter, A. (2005) Sulfur metabolism: a versatile platform for launching defence operations. Trends Plant Sci. 10, 503-509

41 van Roermund, C. W., Elgersma, Y., Singh, N., Wanders, R. J. and Tabak, H. F. (1995) The membrane of peroxisomes in Saccharomyces cerevisiae is impermeable to NAD $(H)$ and acetyl-CoA under in vivo conditions. Embo J. 14, 3480-3486

42 Tilton, G., Shockey, J. and Browse, J. (2000) Two families of acyl-CoA thioesterases in Arabidopsis. Biochem Soc Trans. 28, 946-947

43 Reumann, S., Quan, S., Aung, K., Yang, P. F., Manandhar-Shrestha, K., Holbrook, D., Linka, N., Switzenberg, R., Wilkerson, C. G., Weber, A. P. M., Olsen, L. J. and Hu, J. P. (2009) InDepth Proteome Analysis of Arabidopsis Leaf Peroxisomes Combined with in Vivo Subcellular Targeting Verification Indicates Novel Metabolic and Regulatory Functions of Peroxisomes. Plant Physiology. 150, 125-143

44 Bender-Machado, L., Bauerlein, M., Carrari, F., Schauer, N., Lytovchenko, A., Gibon, Y., Kelly, A. A., Loureiro, M., Muller-Rober, B., Willmitzer, L. and Fernie, A. R. (2004) Expression of a yeast acetyl CoA hydrolase in the mitochondrion of tobacco plants inhibits growth and restricts photosynthesis. Plant Mol Biol. 55, 645-662

45 Carrari, F., Nunes-Nesi, A., Gibon, Y., Lytovchenko, A., Loureiro, M. E. and Fernie, A. R. (2003) Reduced Expression of Aconitase Results in an Enhanced Rate of Photosynthesis and Marked Shifts in Carbon Partitioning in Illuminated Leaves of Wild Species Tomato. Plant Physiology. 133, 1322-1335

46 Fell, D. (1997) Understanding the Control of Metabolism. Portland Press, London

47 Pritchard, S. L., Charlton, W. L., Baker, A. and Graham, I. A. (2002) Germination and storage reserve mobilization are regulated independently in Arabidopsis. Plant Journal. 31, 639-647 48 Cornah, J. E. and Smith, S. M. (2002) Synthesis and function of glyoxylate cycle enzymes. In Plant Peroxisomes (Baker, A. and Graham, I. A., eds.). pp. 57-101, Kluver Academic Publishers, London

49 Allen, E., Moing, A., Ebbels, T. M. D., Maucourt, M., Tomos, A. D., Rolin, D. and Hooks, M. A. (2010) Correlation Network Analysis reveals a sequential reorganization of metabolic and transcriptional states during germination and gene-metabolite relationships in developing seedlings of Arabidopsis. BMC Systems Biology. 4, 62

50 Sheen, J. (1990) Metabolic Repression of Transcription in Higher-Plants. Plant Cell. 2, 1027-1038

51 Penfield, S., Josse, E. M., Kannangara, R., Gilday, A. D., Halliday, K. J. and Graham, I. A. (2005) Cold and light control seed germination through the bHLH transcription factor SPATULA. Current Biology. 15, 1998-2006

52 Fischer, E. and Sauer, U. (2005) Large-scale in vivo flux analysis shows rigidity and suboptimal performance of Bacillus subtilis metabolism. Nat Genet. 37, 636-640 


\section{Figure Legends}

Figure 1. Acetate utilization by acn1-2. Symbols: $\circ$, • Col-7; $\square$, $\mathbf{a c n 1 - 2 . ~ D a r k e n e d ~ s y m b o l s ~}$ represent acetate levels after $\left[2-{ }^{12} \mathrm{C}\right]$ acetate chase. Approximately $1 \mathrm{~g}$ samples of 3 day-old seedlings (PSG 0.7) seedlings were fed with $4 \mathrm{mM}\left[2-{ }^{13} \mathrm{C}\right]$ acetate plus $20 \mathrm{mM}$ sucrose in incubation media for the lengths of time specified. Chase experiments were performed by removing seedlings at $2 \mathrm{~h}$ and transferring them to a solution with $4 \mathrm{mM}$ isotopically normal acetate plus $20 \mathrm{mM}$ sucrose (arrow). Tissue samples were extracted with $80 \%$ ethanol. Acetate was quantified using NMR by comparison of the intensities of the ${ }^{13} \mathrm{C}$ resonances of acetate and the internal standard formate. Symbols and error bars represent the average \pm sd, respectively, of values from 3 independent experiments. According to Student's T-tests, the differences between values pre- and post-chase at $4 \mathrm{~h}$ for both genotypes were significant with $\mathrm{P}<0.01$. There were no significant differences between 4 and $6 \mathrm{~h}$ for post-chase values for either genotype.

Figure 2. Time course quantification of GIn by ${ }^{13} \mathrm{C}$-labelled carbon resonances. Symbols: $\circ$, • Col-7; 口, - acn1-2. Darkened symbols represent measured Gln levels after chasing with [2${ }^{12} \mathrm{C}$ ]acetate chase (arrow). The experimentation is briefly described in the legend to Figure 1. Panels $\mathrm{A}, \mathrm{B}$ and $\mathrm{C}$ refer to the $\mathrm{C} 2, \mathrm{C} 3$ and $\mathrm{C} 4$ positions of $\mathrm{Gln}$, respectively. The values represent the mean \pm sd of 3 independent experiments. According to Student's t-tests, $2 \mathrm{~h}$ pre-chase values between genotypes for all $\mathrm{C}$ positions were not significant with $\mathrm{P}<0.05$. At $4 \mathrm{~h}$, the pre-chase, betweengenotype values were $0.3,0.06$ and 0.07 for $\mathrm{C} 2, \mathrm{C} 3$ and $\mathrm{C} 4$, respectively. All post-chase, betweengenotype values were significant with $\mathrm{P}<0.01$, except for $\mathrm{C} 2$ at $4 \mathrm{~h}(\mathrm{P}=0.054)$.

Figure 3. Degradation of eicosenoic acid. Fatty acids were extracted from seeds at 4 days after imbibing in the cold (day 0 ) and 3 and 5 days after transfer to growth conditions. The values represent means \pm sd of five independent samples. According to Student's t-tests, for both genotypes the levels were significantly different between days with $\mathrm{P}<0.01$, and only at day 3 were the levels of acn1-2 significantly different from those in Col-7 $(\mathrm{P}<0.01)$.

Figure 4. Models to explain the role of ACN1 preventing the loss of carbon from peroxisomes. Abbreviations: Ac, unesterified (free) acetate, Ac-CoA, acetyl-CoA; Cit, citrate, Mal, malate; TE, thioesterase. (A) The wild-type situation showing the anapleurotic/gluconeogenic function of the glyoxylate cycle. The principle flow of carbon from fatty acids out of peroxisomes is through citrate and malate. (B) Removal of ACN1 permits the exit of acetate from peroxisomes due to the action of a thioesterase, where is it reactivated in the cytosol by an acetyl-CoA synthetase activity. 
Table I. Comparison of major metabolite levels in acn1-2 and Col-7.

\begin{tabular}{|c|c|c|c|}
\hline Metabolite $^{a}$ & $\mathrm{Col}-7^{\mathrm{b}}$ & $\operatorname{acn} 1-2^{\mathrm{b}}$ & $P^{\mathrm{c}}$ \\
\hline Alanine & $275 \pm 84$ & $106 \pm 66$ & 0.0036 \\
\hline Arginine & $383 \pm 287$ & $119 \pm 67$ & 0.0541 \\
\hline Glutamate & $2430 \pm 1869$ & $600 \pm 337$ & 0.0469 \\
\hline Glutamine & $9520 \pm 5100$ & $2543 \pm 1298$ & 0.0164 \\
\hline Isoleucine & $30 \pm 14$ & $12 \pm 4$ & 0.0234 \\
\hline Leucine & $158 \pm 60$ & $66 \pm 26$ & 0.0095 \\
\hline Proline & $268 \pm 293$ & $55 \pm 47$ & 0.0906 \\
\hline Threonine & $413 \pm 225$ & $123 \pm 48$ & 0.0216 \\
\hline Valine & $103 \pm 43$ & $36 \pm 15$ & 0.0111 \\
\hline Choline & $471 \pm 202$ & $163 \pm 95$ & 0.0114 \\
\hline Trigonelline & $24 \pm 12$ & $9 \pm 9$ & 0.0199 \\
\hline Fructose & $1165 \pm 601$ & $465 \pm 196$ & 0.0290 \\
\hline Glucose & $1316 \pm 573$ & $522 \pm 318$ & 0.0163 \\
\hline Rhamnose & $29 \pm 20$ & $9 \pm 7$ & 0.0436 \\
\hline Sucrose & $507 \pm 215$ & $193 \pm 98$ & 0.0135 \\
\hline Citrate & $220 \pm 100$ & $135 \pm 59$ & 0.0729 \\
\hline Formate & $160 \pm 80$ & $48 \pm 21$ & 0.0164 \\
\hline Fumarate & $64 \pm 57$ & $75 \pm 68$ & 0.3979 \\
\hline Lactate & $198 \pm 113$ & $68 \pm 10$ & 0.0307 \\
\hline Malate & $465 \pm 220$ & $225 \pm 128$ & 0.0366 \\
\hline Acetyl-CoA & $38 \pm 35$ & $88 \pm 18$ & 0.0200 \\
\hline UnkD8.0 & $10 \pm 6$ & $6 \pm 6$ & 0.1636 \\
\hline UnkM7.9 & $18 \pm 11$ & $6 \pm 6$ & 0.0280 \\
\hline UnkS7.37 & $22 \pm 8$ & $9 \pm 3$ & 0.0099 \\
\hline UnkD5.69 & $93 \pm 52$ & $36 \pm 12$ & 0.0346 \\
\hline UnkM5.18 & $7 \pm 3$ & $3 \pm 3$ & 0.0244 \\
\hline UnkD3.12 & $233 \pm 120$ & $116 \pm 33$ & 0.0407 \\
\hline UnkM1.85 & $108 \pm 61$ & $44 \pm 27$ & 0.0309 \\
\hline
\end{tabular}

${ }^{a}$ The letters and numbers of unknown peaks represent the type of signal and the chemical shift relative to TSP: unk, unknown, D, doublet; $M$, multiplet; S, singlet.

${ }^{\mathrm{b}}$ The number of samples for Col-7 and $a c n 1-2$ were 5 and 6 , respectively.

${ }^{\mathrm{c}}$ The probability statistic $p$ is derived from Student's t-tests with 9 degrees of freedom. 
Table II Comparative gene expression of Col-7 and acn1-2 from transcript profiling. The 20 greatest up-and down-regulated genes and acetate assimilation genes with fold-DE greater than 1.4 are shown. The gene descriptions are those given in TAIR10 Genome Release. Fold-DE, fold differential expression (ratio of intensity values).

\section{Gene ID \\ Gene Description \\ Up-regulated in acn1-2}

Fold-DE

At4g31070

At3 849460

At1 g49920

At1 g56680

At5g13350

At1 g22080

At1 27590

At5g19920

At2g04620

At2g46780

At3g28510

At1 g75230

At2g17920

At1 g66360

At2g06480

At3g19330

At5g24655

At4g18780

At5g25950

At4g05240

At5g56720

At1 g08650

At2g36640

At1 g33055

At1 g72070

At1 g48660

At1 g32560

At4g36600

At2g33850

At4g21020

At5g28630

Atlg10640
Tetratricopeptide repeat (TPR)-like superfamily protein

Unknown protein

MuDR family transposase

Chitinase family protein

21.2

Auxin-responsive GH3 family protein

19.6

Cysteine proteinases superfamily protein

16.7

16.0

14.0

14.0

13.7

12.7

11.2

10.2

9.4

9.0

9.0

8.4

8.1

7.5

7.5

Ubiquitin-like superfamily protein

Lactate/malate dehydrogenase family protein

Phosphoenolpyruvate carboxylase kinase

\section{Down-regulated in acn 1-2}

Late embryogenesis abundant (LEA) protein in group 3

52.8

Unknown protein

45.0

Chaperone DnaJ-domain superfamily protein

38.4

Auxin-responsive GH3 family protein

34.4

30.6

Late embryogenesis abundant protein, group 1 protein

28.5

24.9

Unknown protein

24.9

Glycine-rich protein

23.1

21.7 
$\begin{array}{lll}\text { At5g62210 } & \text { Embryo-specific protein 3, (ATS3) } & 20.1\end{array}$

$\begin{array}{lll}\text { At1g33265 Transmembrane proteins 14C } & 19.1\end{array}$

$\begin{array}{lll}\text { At1g24090 RNase H family protein } & 17.0\end{array}$

At4g35480 Encodes a putative RING-H2 finger protein RHA3b. 16.5

$\begin{array}{lll}\text { At3g45460 IBR domain containing protein } & 16.0\end{array}$

$\begin{array}{llr}\text { At4g36830 HOS3-1 } & 15.5\end{array}$

At3g02480 Late embryogenesis abundant protein (LEA) family protein

At4g20800 FAD-binding Berberine family protein 14.9

At2g32790 Ubiquitin-conjugating enzyme family protein 14.6

At5g39110 RmlC-like cupins superfamily protein $\quad 14.3$

\section{Acetate Assimilation Genes}

At5g03860 Encodes a protein with malate synthase activity. $\quad 5.5$

At3g21720 Encodes a glyoxylate cycle enzyme isocitrate lyase (ICL) 4.3

At3g53910 malate dehydrogenase-related 3.5

At2g42790 Peroxisomal citrate synthase 1.7

$\begin{array}{lll}\text { At3g16910 ACN1/AAE7 } & 1.5\end{array}$

At2g30970 Aspartate aminotransferase 1 1.4 
Figure 1.

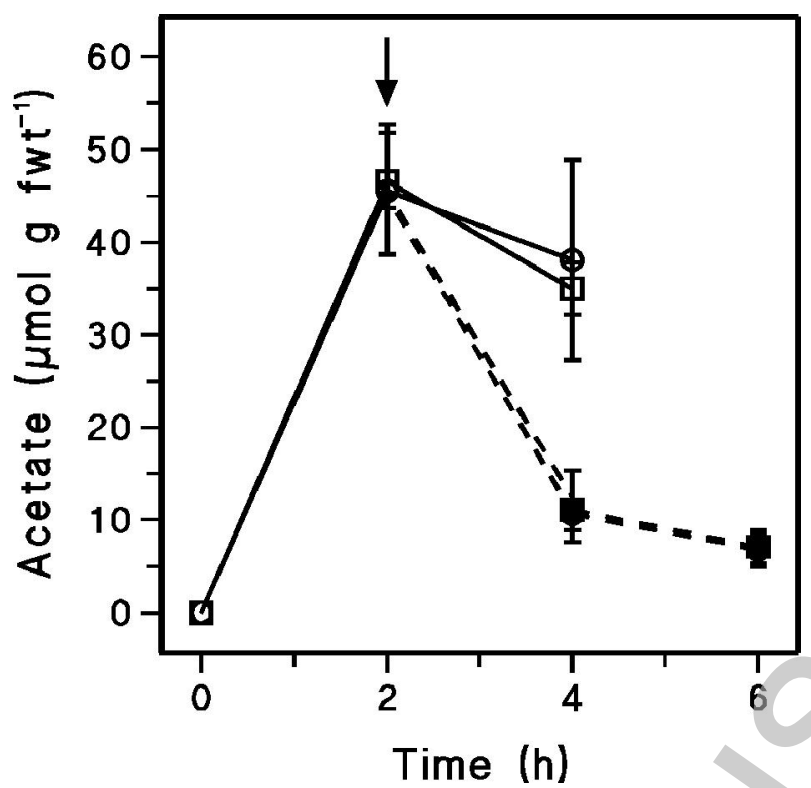

Figure2

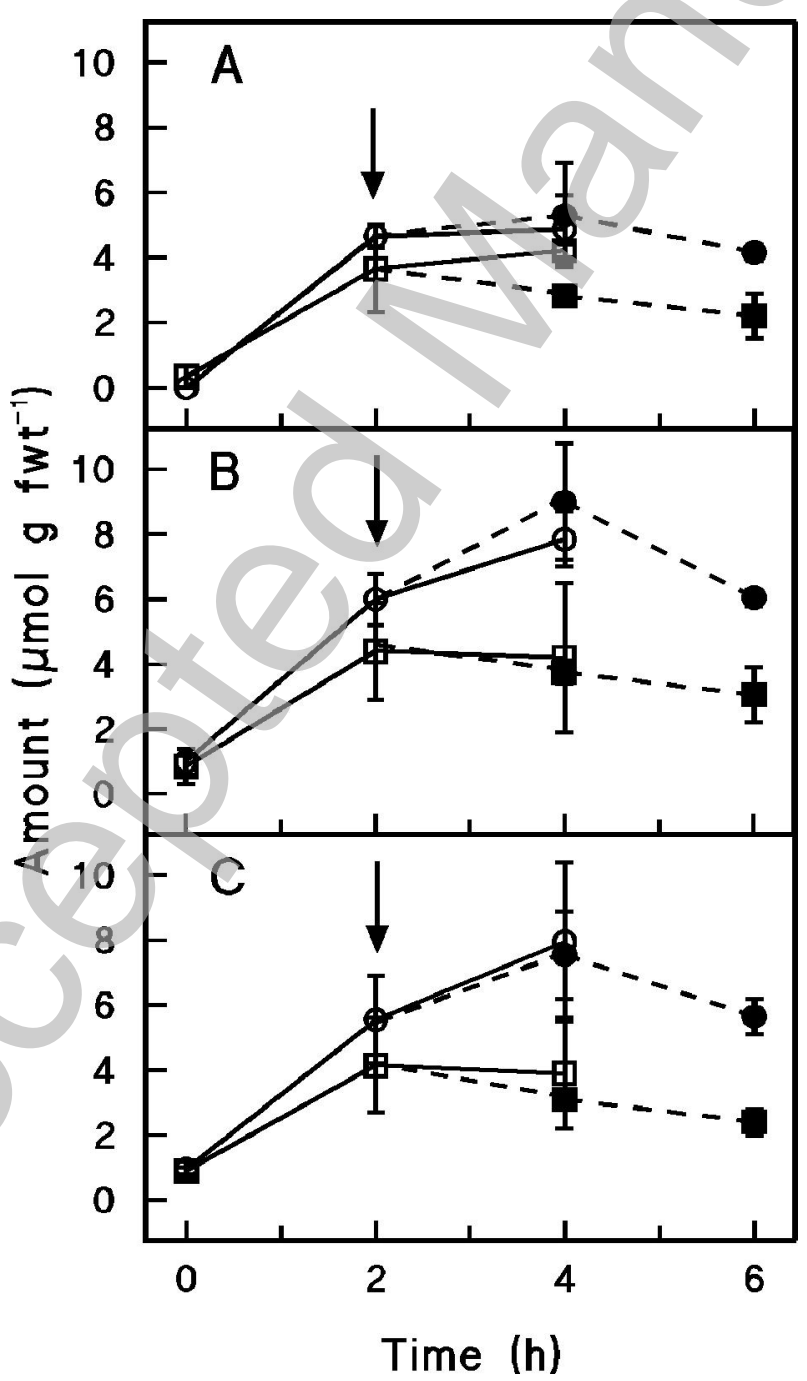


Figure 3.

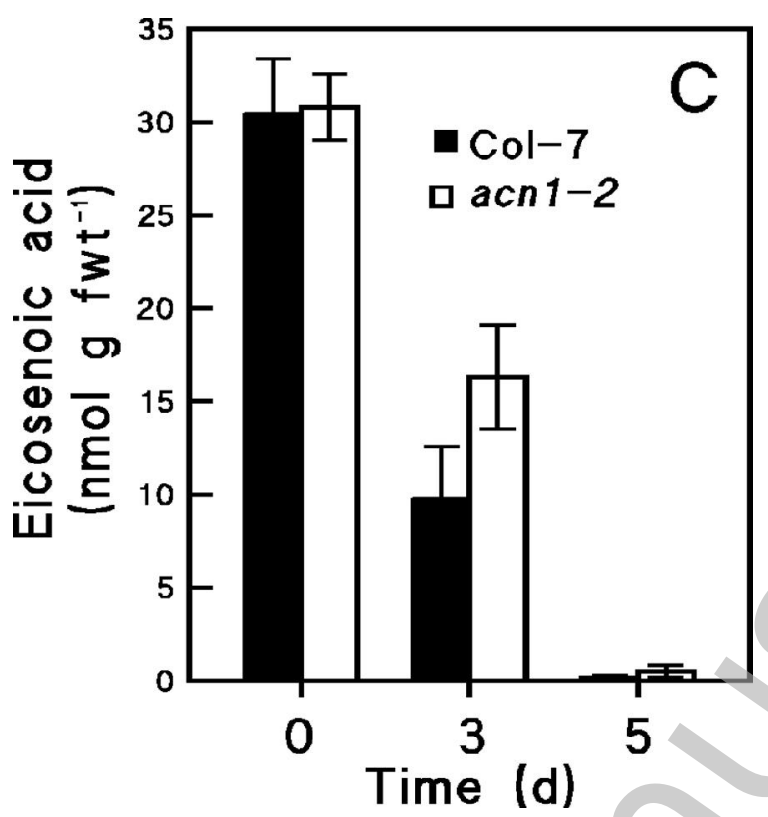

Figure 4.
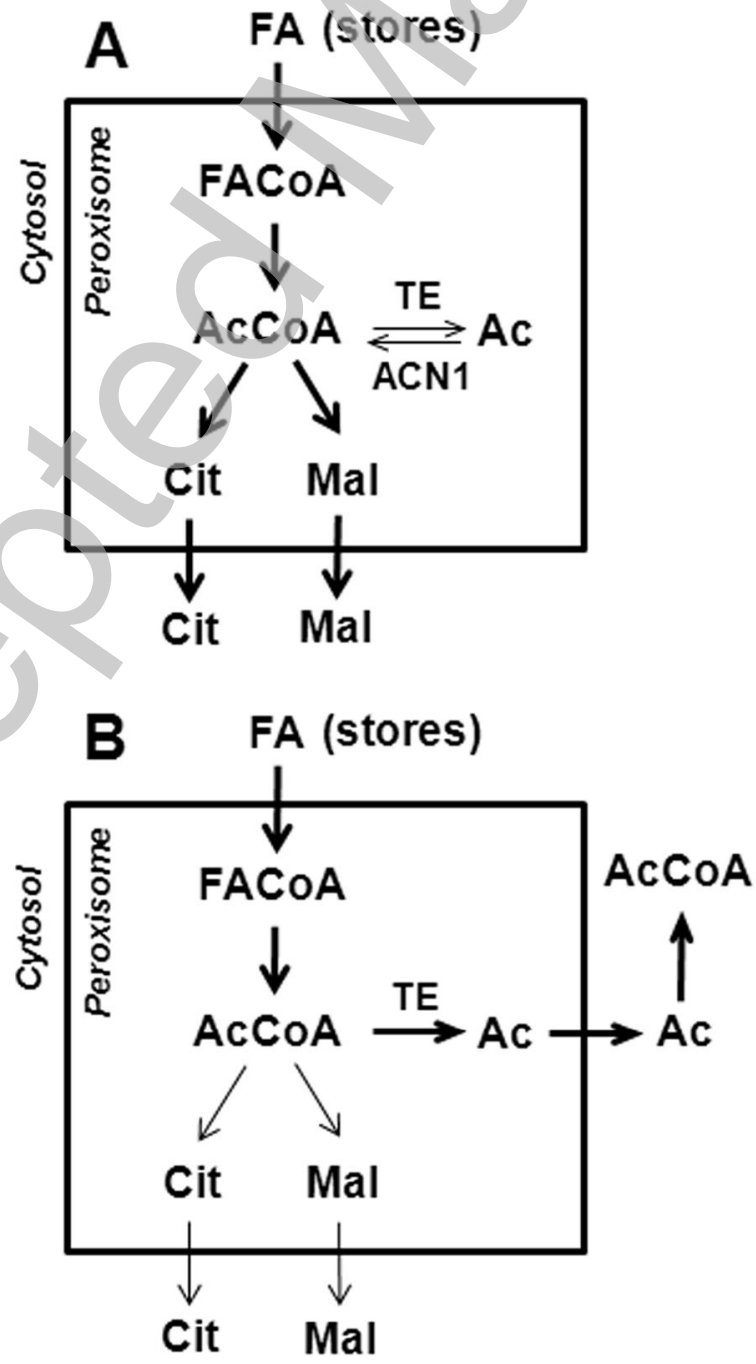

-19 -

Licenced copy. Copying is not permitted, except with prior permission and as allowed by law. (C) 2011 The Authors Journal compilation (c) 2011 Portland Press Limited 